\title{
Metabolic competition between lipid metabolism and histone methylation regulates sexual differentiation in human malaria parasites.
}

Chantal T. Harris ${ }^{1,2}$, Xinran Tong ${ }^{1,3}$, Riward Campelo ${ }^{1}$, Leen N. Vanheer ${ }^{1}$, Inês M. Marreiros ${ }^{4,5}$, Navid Nahiyaan ${ }^{6}$, Vanessa A. Zuzarte-Luís ${ }^{4}$, Kirk W. Deitsch ${ }^{1}$, Maria M. Mota ${ }^{4}$, Kyu Y. Rhee ${ }^{1,6}$, Björn F.C. Kafsack ${ }^{1, *}$

${ }^{1}$ Department of Microbiology \& Immunology, Weill Cornell Medicine, New York, USA.

${ }^{2}$ Immunology \& Microbial Pathogenesis Graduate Program, Weill Cornell Medicine, New York, USA.

${ }^{3}$ BCMB Allied Graduate Program, Weill Cornell Medicine, New York, NY 10065, USA.

${ }^{4}$ Instituto de Medicina Molecular João Lobo Antunes, Faculdade de Medicina Universidade de Lisboa, Lisbon, Portugal.

${ }^{5}$ Instituto de Ciências Biomédicas Abel Salazar (ICBAS), Universidade do Porto, Porto, Portugal.

${ }^{6}$ Division of Infectious Diseases, Weill Department of Medicine, Weill Cornell Medicine, New York, USA.

* Please send correspondence to bjk2007@med.cornell.edu

\section{Abbreviations}

3-DZA: 3-deaza-adenosine; cho: choline; CDP-cho: cytidine diphosphate-choline; GlcNAC: N-acetyl glucosamine; Glm: glucosamine; hcys: homocysteine; $R B C$ : infected RBC; lysoPC: lyso-phosphatidylcholine; met: methionine; P-cho: phospho-choline; P-etn: phospho-ethanolamine; P-etn-me1/2: mono/dimethylphosphotehanolamine; PMT: phosphoethanolamine methyltranserase; RBC: red blood cell; SAMS: Sadenosylmethionine synthetase; SAHH: S-adenosylhomocysteine hydrolase; SAH: S-adenosyl homocysteine; SAM: S-adenosylmethionine; ser: serine; TMP: trimethoprim; $U R B C$ : uninfected RBC. 


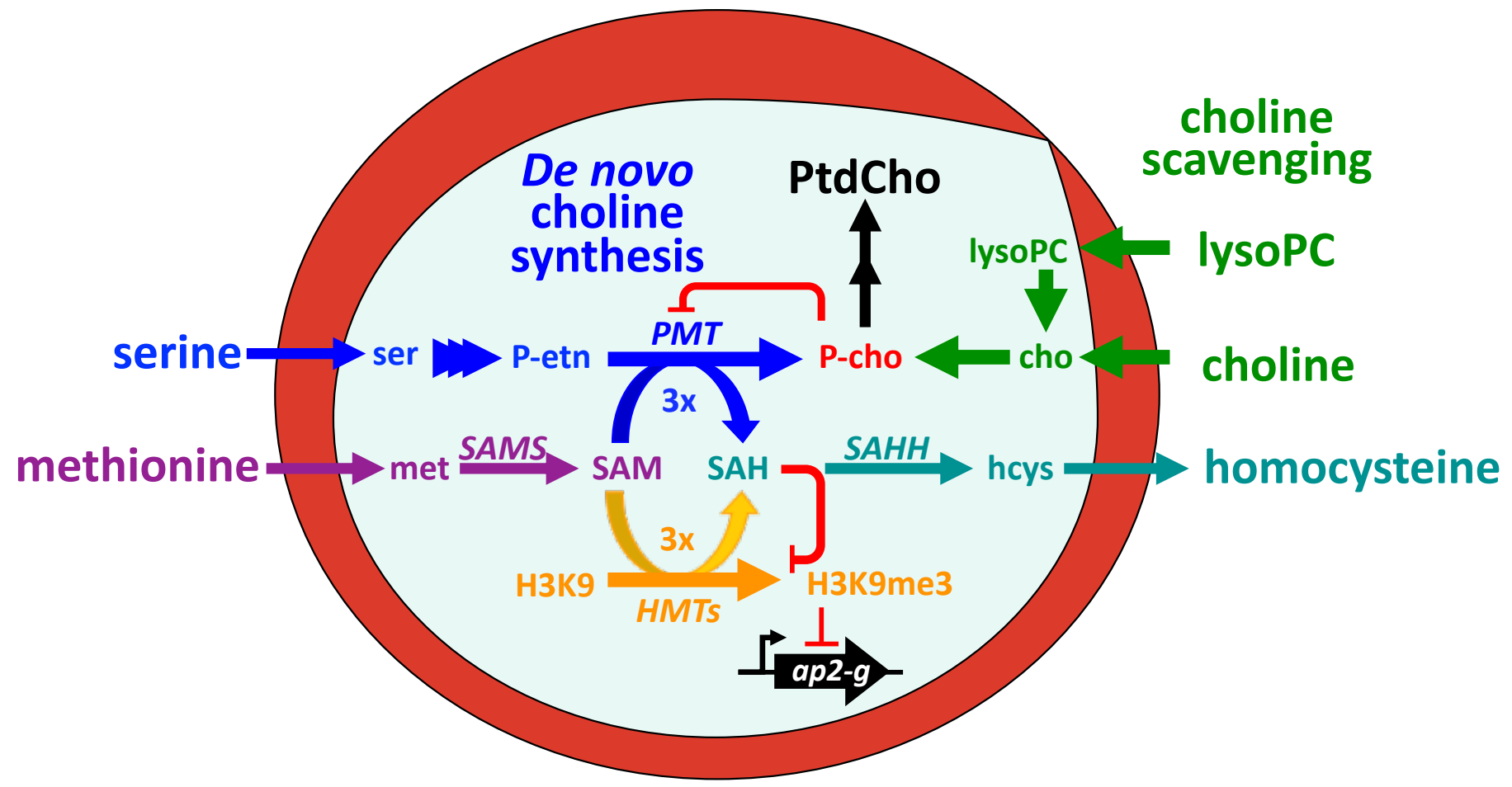

\section{ABSTRACT}

Like most pathogens, malaria parasites balance persistence in the current host with transmission to the next. For Plasmodium falciparum, the most widespread and virulent human malaria parasite, persistence depends on continuous asexual replication in red blood cells while transmission requires differentiation into non-replicating gametocytes, the male and female cells able to infect the mosquito vector. This developmental decision is controlled via stochastic derepression of a heterochromatin-silenced locus encoding the transcription factor PfAP2-G, which acts as the master switch for sexual differentiation. Recent work showed that the frequency of pfap2-g derepression is responsive to extracellular levels of phospholipid precursors. However, the regulatory mechanisms linking these metabolites to epigenetic regulation of pfap2-g was hitherto unknown. Using chemical, metabolomic, and genetic approaches, we show that this response is mediated by metabolic competition for the methyl donor S-adenosylmethionine between histone methyltransferases and phosphoethanolamine methyltransferase, a critical enzyme in the parasite's pathway for de novo phosphatidyl-choline synthesis. When phosphatidyl-choline precursors are scarce, increased consumption of S-adenosylmethionine for de novo synthesis of phosphatidyl-choline results in a decrease in histone methylation marks that mediate silencing of pfap2-g, thereby up-regulating its transcription and increasing the frequency of sexual differentiation. Our findings reveal a key connection between metabolite utilization and gene expression in malaria parasites that forms the mechanistic link between phosphatidyl-choline metabolism and the frequency of sexual commitment. 


\section{INTRODUCTION}

Transmission of malaria parasites requires differentiation from replicating asexual blood-stream forms that are responsible for pathogenesis into non-replicating sexual stages, called gametocytes, that can infect the mosquito vector. Parasite success depends on a careful balance between asexual replication, to maintain human infection, and gametocyte formation, to infect the mosquito vector for further transmission. The regulation of this balance is key to understanding the dynamics of malaria pathogenesis and transmission(1). Developmental commitment to sexual differentiation requires expression of the transcription factor AP2-G(2, 3). This requires the transcriptional activation of the ap2-g locus, which is actively silenced in a heterochromatin-dependent manner during asexual replication(2, 4-6). The frequency of sexual differentiation relies on the efficiency of heterochromatin maintenance at the pfap2-g locus, which depends on the efficient trimethylation of lysine 9 on histone $\mathrm{H} 3$ of newly placed nucleosomes and recognition of this mark by heterochromatin protein $1(2,5,7$, 8). As long as ap2-g is efficiently silenced, parasites continue replicating asexually. However, when heterochromatin maintenance at this locus is impaired, low-level AP2-G expression can activate a transcriptional feedback loop when the transcription factor binds motifs upstream of its own locus, upregulating its transcription. This drives AP2-G expression to high levels and activates the transcriptional program underlying gametocytogenesis(2, 9-12).

In the laboratory, the frequency of sexual commitment of Plasmodium falciparum, the most wide-spread and virulent human malaria parasite, varies from less than $1 \%$ to greater than $40 \%$ in response to culture conditions, including parasite density and media composition(13). Recent work showed that lysophosphatidylcholine (lysoPC) and choline, precursors of phosphocholine (P-cho) headgroup required for phosphatidylcholine (PtdCho) synthesis, act as potent suppressors of sexual commitment(14). This ability of parasites to vary their investment into gametocyte production illustrates the parasites' ability to sense their inhost environment and respond adaptively to resource abundance(15) or anatomical niche(16, 17). However, the mechanisms underlying this response, that is, link the availability of these metabolites to $p f a p 2-g$ activation remain unknown. Here, we show that the availability of P-cho precursors regulates sexual commitment by shifting the metabolic competition for the methyl donor S-adenosyl methionine (SAM) between the methyltransferase required for de novo synthesis of P-cho and the histone methyltransferases maintaining heterochromatin-mediated silencing at the ap2-g locus.

\section{P-cho precursor availability alters sexual commitment and intracellular concentrations of SAM and SAH.}

As the parasite grows during its 48 -hour asexual replication cycle, the membrane content of infected red blood cells (iRBCs) increases 8-fold, with PtdCho accounting for more than half of this increases in membrane biomass $(18,19)$. In P. falciparum, PtdCho is exclusively derived from P-cho, which can be generated de novo 
bioRxiv preprint doi: https://doi.org/10.1101/2022.01.18.476397; this version posted January 18, 2022. The copyright holder for this preprint (which was not certified by peer review) is the author/funder, who has granted bioRxiv a license to display the preprint in perpetuity. It is made available under aCC-BY-NC 4.0 International license.

from serine via phospho-ethanolamine (P-etn) or scavenged from extracellular sources, including choline and choline-containing phospholipids such as lysoPC(20) (Figure 1A). When P-cho precursors are available, parasites down-regulate phospho-ethanolamine methyltranserase (PMT), which generates P-cho by transferring three consecutive methyl-groups from the methyl-donor S-adenosylmethionine (SAM) onto P-etn(20, 21).

Consistent with earlier reports(14), we found that supplementation with exogenous P-cho precursors during the a single replication cycle (commitment cycle) suppressed sexual commitment(Figure 1B-C) and increased the P-cho and CDP-choline levels of iRBCs compared to conditions when these precursors where scarce (Figure 1D). As in other eukaryotes(22), de novo synthesis of PtdCho is also a major consumer of SAM and source of S-adenosyl homocysteine $(\mathrm{SAH})$ in malaria parasite blood-stages. When exogenous $\mathrm{P}$-cho precursors are abundant, levels of SAM increase and SAH levels decrease compared to conditions requiring de novo synthesis of P-cho from P-etn (Figure 1E). This response is dose dependent (Figure S1) indicating that Pcho precursor availability regulates intracellular levels of SAM and SAH. These changes in the intracellular abundances of P-cho, SAM, and SAH were limited to iRBCs and absent from uninfected RBCs (Figure S2).
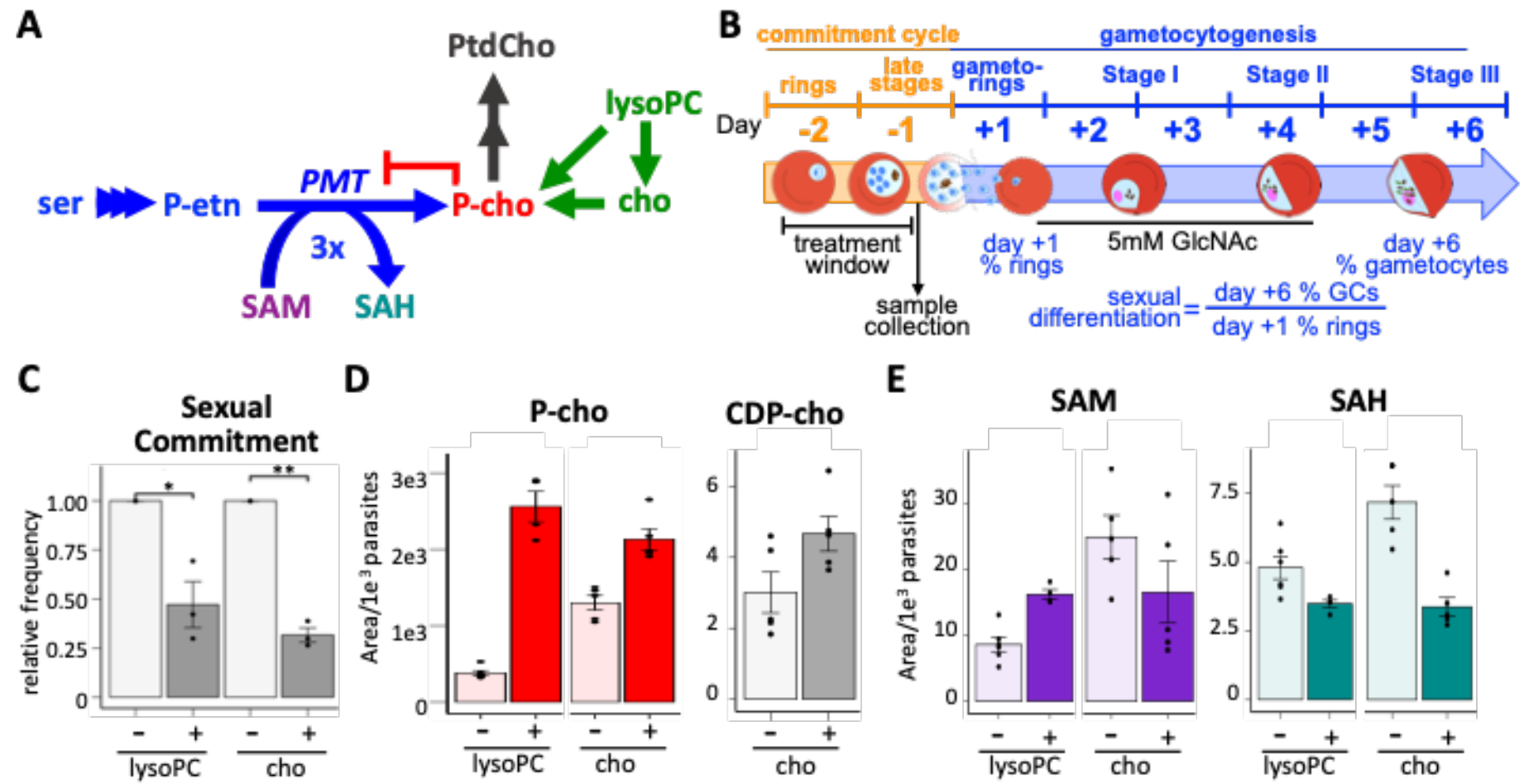

Figure 1. Phospho-choline precursor availability alters parasite SAM and SAH levels.

(A) PtdCho is generated exclusively from P-cho, which can be scavenged from extracellular choline or lysoPC or synthesized de novo via triple methylation of P-etn by PMT, consuming 3 SAM and producing 3 SAH per P-cho. (B) Synchronous blood-stages were grown for a single cycle (commitment cycle) under various nutrient conditions and samples were collected 36 hours post-invasion. Treated parasites were allowed to re-invade and $50 \mathrm{mM} \mathrm{N}$-acetylglucosamine was added on Day +1 to block asexual replication. The sexual differentiation rate is defined as the percentage of Day +1 ring stages that differentiate into stage III gametocytes by Day +6 . (C) $P$ cho precursors inhibit the frequency of sexual differentiation. Bars indicate sexual commitment rates relative to conditions without P-cho supplementation. ( $n=3$ ) (D-E) Intracellular metabolite levels are altered in response to lysoPC or choline supplementation in parasite media. Bars indicate the calculated area under the signal curve for each metabolite per $1 e^{3}$ parasites $(n=3)$. Bar graphs show the mean \pm s.e.m. ${ }^{*} \mathrm{p}<0.05,{ }^{* *} \mathrm{p}<0.01$, for paired two-sided t-test. 
bioRxiv preprint doi: https://doi.org/10.1101/2022.01.18.476397; this version posted January 18,2022 . The copyright holder for this preprint (which was not certified by peer review) is the author/funder, who has granted bioRxiv a license to display the preprint in perpetuity. It is made available under aCC-BY-NC 4.0 International license.

\section{PfPMT activity drives changes in intracellular levels of SAM and SAH.}

In the presence of P-cho precursors, the need for de novo synthesis is reduced and parasites down-regulate pfpmt(23) as illustrated by increases in P-cho along with decreases in pfpmt transcript levels (Figure 2A) and levels of the mono- and di-methylated P-etn reaction intermediates (Figure 2B). If P-cho synthesis by PfPMT is a major metabolic sink of SAM and source of SAH as hypothesized, their levels should track PfPMT abundance and activity. Quantitation of these metabolites in pfpmt knockout parasites(24) indeed showed a nearly 5-fold increase in intracellular SAM along with SAH levels falling below the level of detection when compared to wildtype parasites (Figure 2C). Intriguingly, a near complete suppression of gametocytogenesis was observed in pfpmt knockout parasites that was restored to parental levels upon genetic complementation(24). Genetic complementation also restored SAM to wild-type levels and increased SAH substantially. These data suggest that PfPMT activity is a significant regulator of intracellular SAM/SAH levels, as well as gametocytogenesis.

To confirm this connection between PfPMT activity, SAM metabolism and sexual commitment, we generated inducible pfpmt knockdown parasites by inserting the glucosamine-responsive autocatalytic glmS
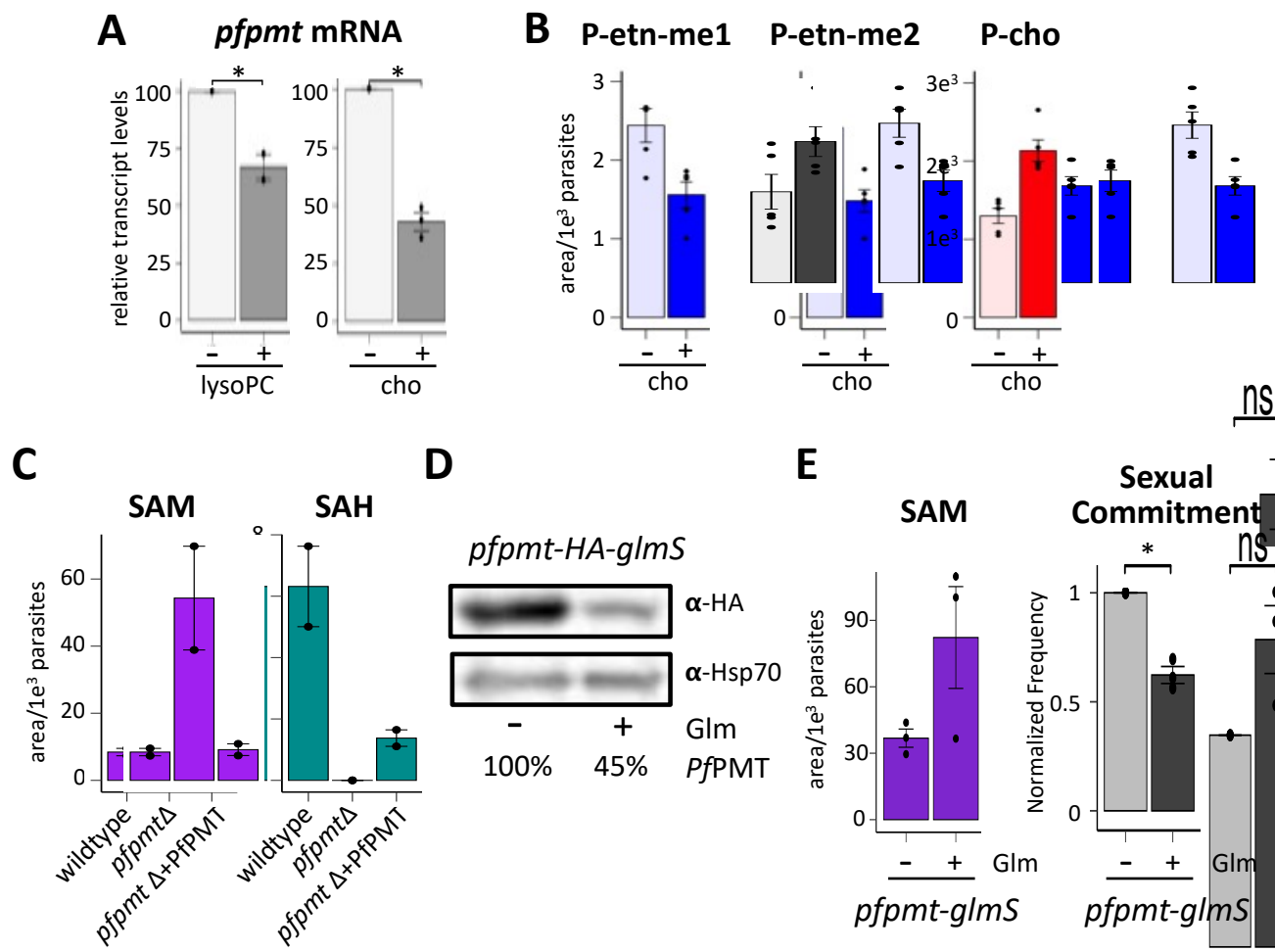

Figure 2. De novo synthesis of P-cho by PMT is a major sink of SAM and source of SAH.

(A) Down-regulation of pmt transcript abundance upon supplementation with P-cho precursors lysoPC and choline (cho). Abundance was normalized to seryl-tRNA synthetase transcript and shown relative to no P-cho precursor supplementation. $\mathrm{n}=2-3$ (B) Choline supplementation results in increased P-cho by scavenging but reduces PMT activity as indicated by reduced levels of the mono- and di-methyl-phospho-ethanolamine intermediates. Metabolites were quantified by LC-MS with bars graphs indicating the area under the curve per thousand parasites $(n=4)$. (C) Loss of PMT (pfpmt $\Delta$ ) results in drastic changes in intracellular SAM and SAH levels that are restored after complementation (pfpmt $\Delta+P f P M T)$. ( $n=2)$. (D) Inducible knockdown of PMT upon addition of $2.5 \mathrm{mM}$ glucosamine $(\mathrm{Glm})$ in PfPMT-gImS parasites significantly reduces PMT protein abundance relative to PfHSP70 loading control. $p<0.05$, student's t test $(n=3)$ (E) Knockdown of PMT increases intracellular SAM levels and reduces sexual commitment even in the absence of P-cho precursors (right panel). Bar graphs show the mean \pm s.e.m. ${ }^{*} p<0.05$ for paired two-sided t-test. 
ribozyme into the endogenous pfpmt 3'UTR (25) (Figure S3A). Treatment of pfpmt-glmS cultures with glucosamine during the commitment cycle reduced PfPMT protein levels by 55\% (Figure 2D, Figure S3C), resulting in a 2-fold increase in intracellular SAM and significantly suppressed sexual commitment, even in the absence of P-cho precursors (Figure 2E-F).

\section{Intracellular SAM and SAH levels both regulate sexual commitment.}

To determine whether these changes in SAM and SAH abundance merely correlate with PfPMT activity or directly regulate sexual commitment, we set out to manipulate their intracellular concentrations independently of PfPMT activity and P-cho precursor abundance. We reasoned that if the availability of P-cho precursors suppresses sexual commitment by reducing PfPMT's consumption of SAM, then this suppression should depend on overall SAM availability. In malaria parasites, SAM is generated solely by SAM synthetase (SAMS) from methionine. Malaria parasites are methionine auxotrophs and, unlike most eukaryotes, lack orthologs to enzymes able to regenerate methionine from homocysteine(26), making parasite SAM synthesis fully dependent on methionine derived from host cell proteins or extracellular pools (Figure 3A). Removing extracellular methionine during a single commitment cycle substantially decreased intracellular methionine and SAM but had no discernable effect on parasite replication (Figure 3B, Figure S4). In standard growth medium containing $100 \mu \mathrm{M}$ methionine, choline significantly suppressed commitment, but this suppressive effect was negated when methionine was removed from the growth media (Figure 3C).

Since methionine is critical for a variety of cellular functions including translation, we generated glucosamine-regulatable pfsams-glmS knockdown parasites to evaluate whether this effect was specific to SAM availability (Figure S5). Upon addition of glucosamine, protein levels of PfSAMS were reduced by 58\% (Figure 3D), which, even in the presence of choline, resulted in a two-fold reduction in SAM levels (Figure 3E) along with a more than 2.5-fold increase in sexual commitment (Figure 3F). Together, these findings demonstrate that sexual commitment is directly regulated by SAM availability.

Each transfer of a methyl group from SAM generates one equivalent of SAH, which can act as a potent feedback inhibitor for many SAM-dependent methyltransferases(27). De novo P-cho synthesis by PfPMT therefore represents not only a major sink of SAM but also an equivalently large source of intracellular SAH (Figure 2, Figure S1). To test whether direct changes to the intracellular levels of SAH alter sexual commitment, we treated parasites during the commitment cycle with 3-deaza-adenosine (3-DZA), a potent inhibitor of SAH hydrolase (SAHH), the enzyme that converts SAH into adenine and homocysteine (Figure 3G)(28, 29).

Addition of 100nM 3-DZA during the commitment cycle, increased intracellular SAH 5-fold (Figure 3H) and more than doubled sexual commitment (Figure 3I), fully reversing the suppressive effects of choline supplementation. These results demonstrate that $\mathrm{SAH}$, in addition to SAM availability, regulates sexual 
A

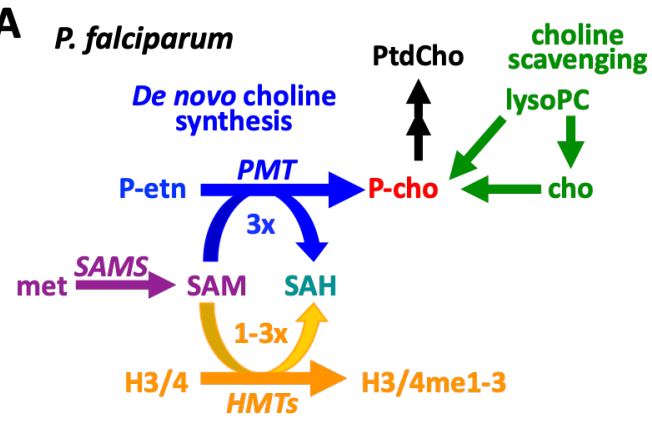

\section{B}

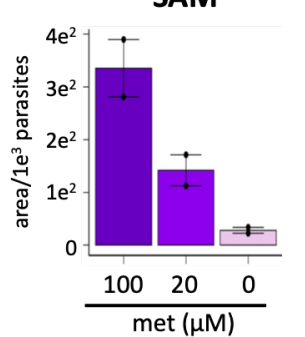

C

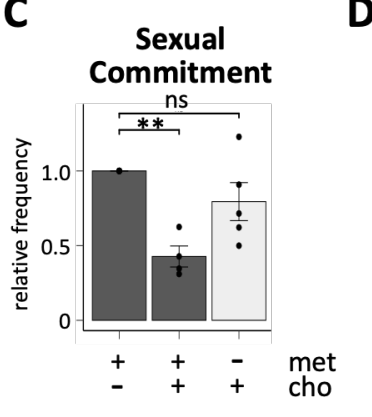

D

\section{pfsams-glms}

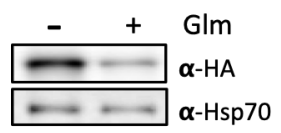

$100 \% 42 \%$ PFSAMS
$\mathbf{E}$

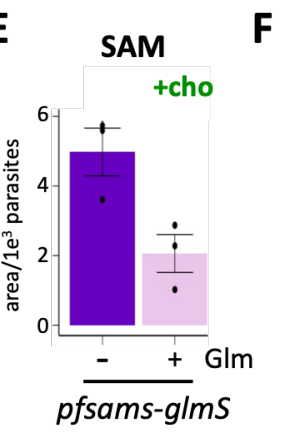

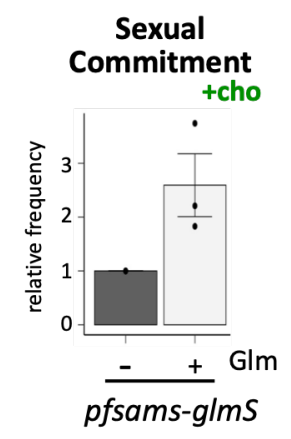

$\mathbf{G}$

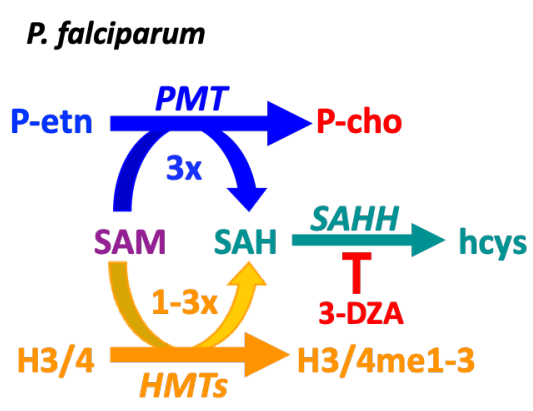

H

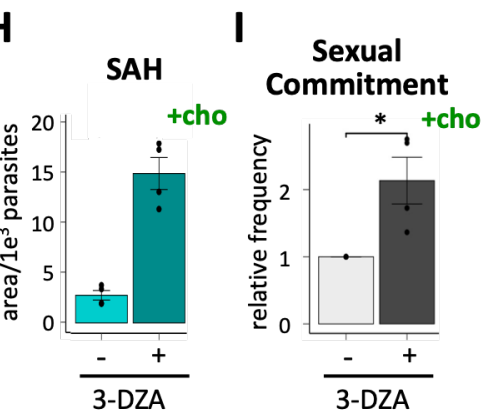

J. berghei

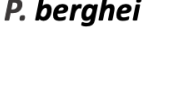

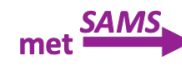

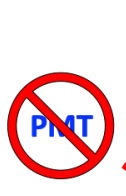
PtdCho choline

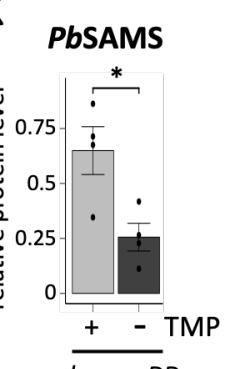

\section{Sexual} Commitment

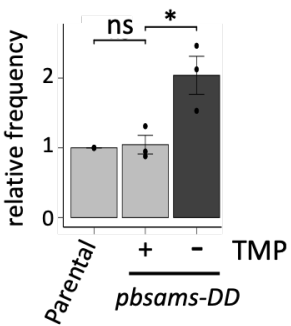

Figure 3. Intracellular SAM and SAH abundance regulate the frequency of sexual differentiation.

(A) SAM and SAH are substrates and product of both de novo P-cho synthesis and histone methylation. (B) Intracellular SAM abundance in growth medium containing standard $(100 \mu \mathrm{M})$ or reduced levels of methionine $(20$ or $0 \mu \mathrm{M})$. ( $n=2)$ (C) Removal of extracellular methionine reverses the suppressive effects of choline supplementation ( $n=4)$. (D-F) Knockdown of PfSAMS in pfsams-HA-glmS parasites by treatment with $2.5 \mathrm{mM}$ glucosamine (Glm) (D) reduced intracellular SAM by 59\% (E) and increased the sexual differentiation rate 2.5-fold (F). (G-I) Treatment with $100 \mathrm{nM}$ 3-DZA increased intracellular SAH 5.4-fold and more than doubled the rate of sexual differentiation even in the presence of P-cho precursors. $(n=3)$. (J) Loss of pmt in rodent malaria parasites, including $P$. berghei, decouples SAM and SAH from PtdCho synthesis. (K) Removal of the stabilizing ligand TMP from P. berghei PbSAMS-DD-HA knockdown parasites reduced PbSAMS by 61\% and (L) resulted in a 2-fold increase in sexual differentiation. Bar graphs show the mean \pm s.e.m. ${ }^{*} p<0.05,{ }^{* *} p<0.01, n s>0.05$ for paired two-sided t-test. 
commitment rates. These data are in alignment with a previous study which found that homocysteine, the metabolic product and subsequent inhibitor of SAH hydrolase activity that accumulates in culture and in the serum of patients and animals infected with malaria parasites, induces gametocytogenesis in culture (30).

\section{SAM but not P-cho precursor levels regulate sexual commitment in rodent malaria parasites.}

Unlike in P. falciparum, supplementation with P-cho precursors had little to no effect on sexual commitment in the rodent malaria parasite Plasmodium berghei(14). In the context of our proposed model, where the consumption of SAM by PfPMT provides the link between P-cho precursors and histone methylation, this observation is readily explained by the fact that the pfpmt ortholog was lost in the rodent malaria parasite lineage, thereby decoupling P-cho availability from SAM and SAH abundance in rodent parasites (Figure 3J). However, since heterochromatin-mediated silencing of pfap2-g also controls sexual commitment in rodent parasites (3), we hypothesized that commitment in $P$. berghei would never-the-less remain sensitive to changes in SAM availability. To test this, we generated SAMS knockdown parasites in $P$. berghei by creating a C-terminal fusion of the endogenous coding sequence with the ecDHFR-based destabilization domain (pbsams-dd-ha, Figure S6). Upon removal of the stabilizing ligand trimethoprim (TMP), PfSAMS expression was reduced by $60 \%$ in pbsams-dd-ha blood-stages (Figure 3K) and resulted in a two-fold increase in sexual commitment (Figure 3L). This demonstrates that SAM availability regulates the rate of sexual commitment even when decoupled from PtdCho metabolism.

\section{Intracellular SAM and SAH level regulate heterochromatin maintenance at the pfap2-g locus.}

In other eukaryotic systems, histone methylation dynamics and gene regulation are responsive to changes in SAM and SAH metabolism(22, 31-34). Methionine depletion and reduction in SAM abundance lead to decreased methylation of specific histone modifications including H3K4me3, H3K9me3, H3K4me2, and H3K36me3, with H3K4me3 exhibiting the most profound changes and leading to a changes in gene expression(22, 31, 35). Since changes in intracellular SAM and SAH directly alter commitment rates (Figure 3), we tested whether these changes could substantially alter the abundance or distribution of the main histone methylation marks associated with silencing (H3K9me3) and activation (H3K4me3) of pfap2-g (Figure 4)(36, 37). To evaluate the effect of SAM on histone methylation patterns we compared the distribution of these marks in the presence of abundant extracellular choline and methionine when SAM levels are high to conditions of low intracellular SAM, when those metabolites were absent from the growth medium (Figure 4A, top right panel). In contrast to H3K4me3, which was slightly elevated when intracellular SAM was low, we observed genome-wide reductions in the abundance of the repressive H3K9me3 mark (Figure 4A-B) under low SAM conditions when compared to high SAM conditions. This reduction in $\mathrm{H} 3 \mathrm{~K} 9 \mathrm{me} 3$ was observed in regions of sub-telomeric heterochromatin as well as for non-subtelomeric heterochromatin islands. 
A Low vs. High SAM $\quad \triangle H 3 K 4 m e 3$
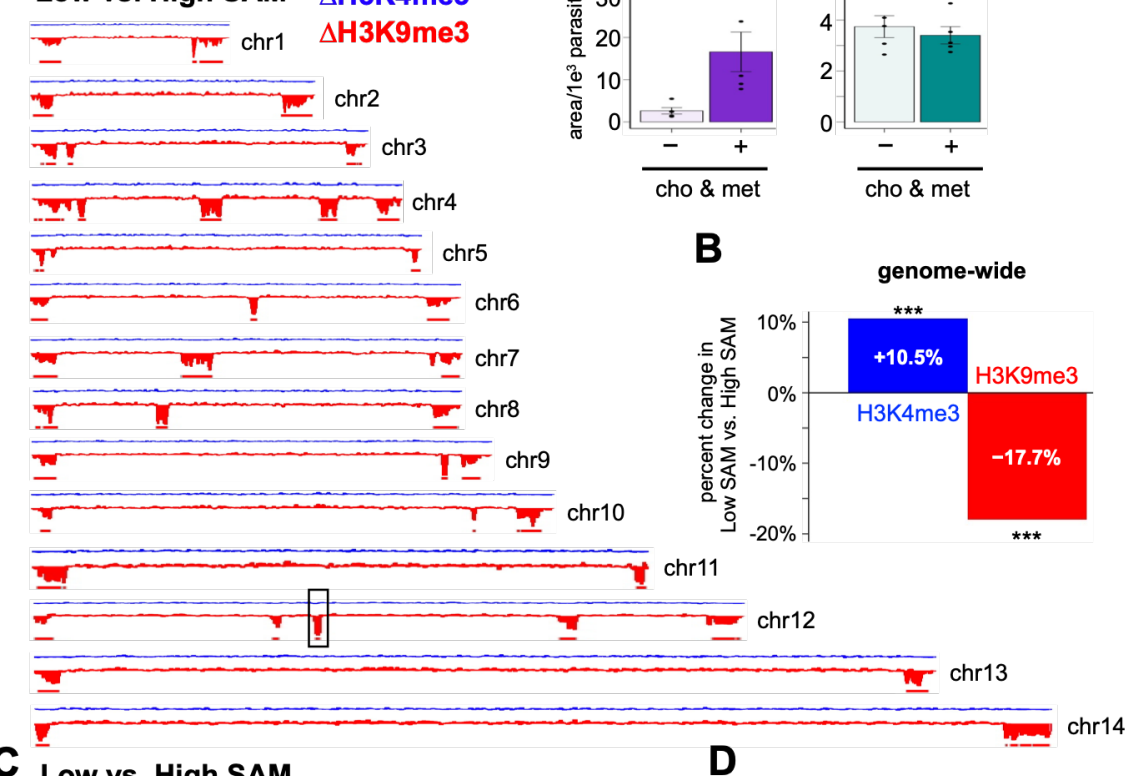

C Low vs. High SAM

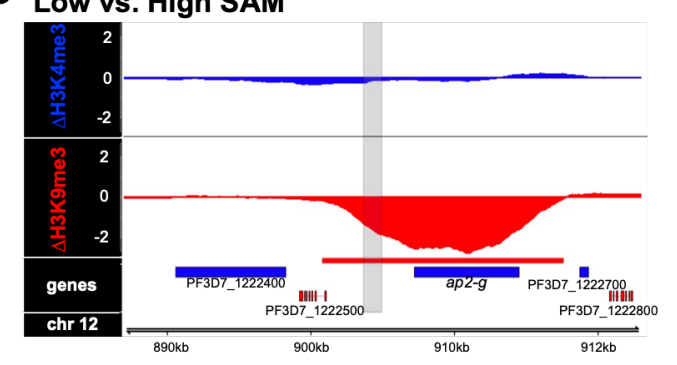

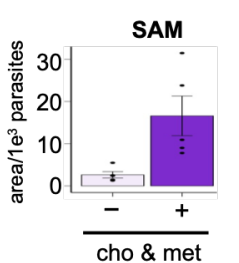

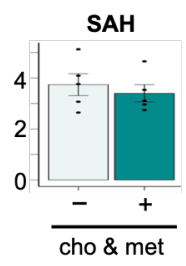

B

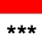

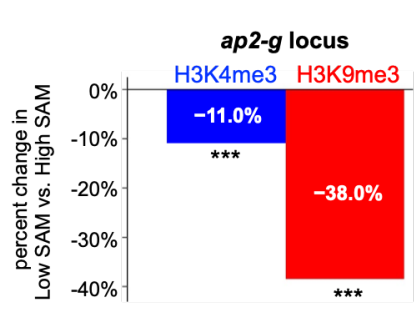

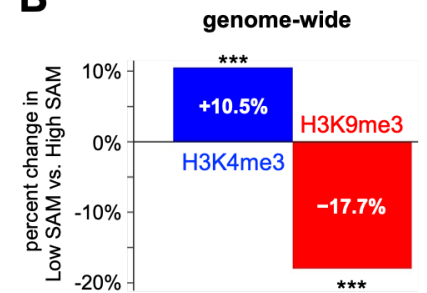

E

High vs. Low SAH $\triangle H 3 K 4 m e 3$
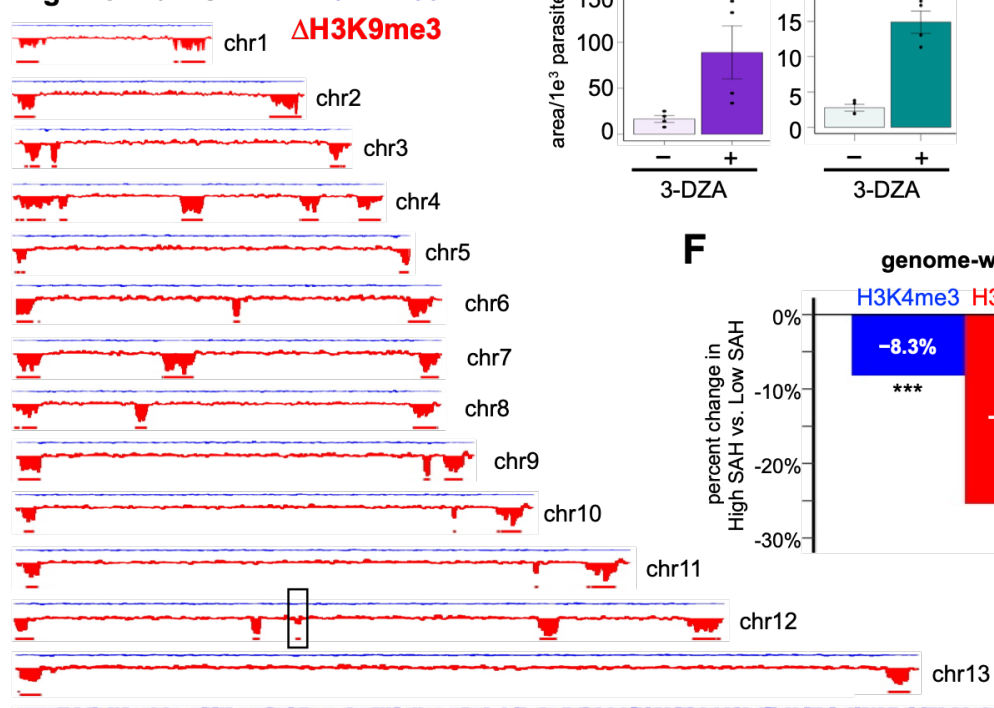

G
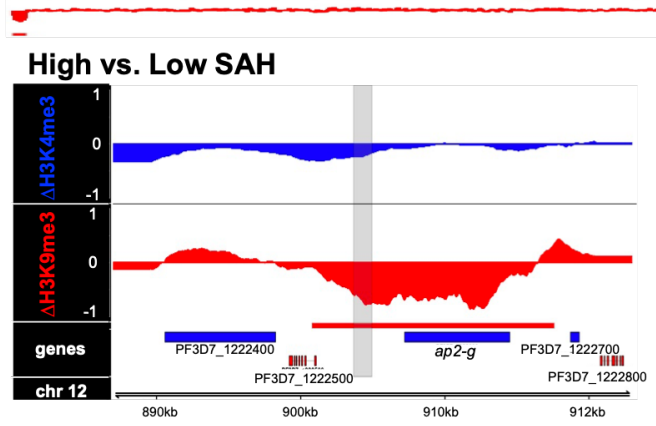

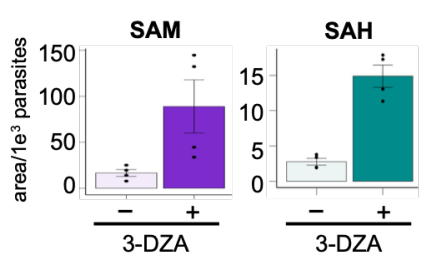

$\mathbf{F}$

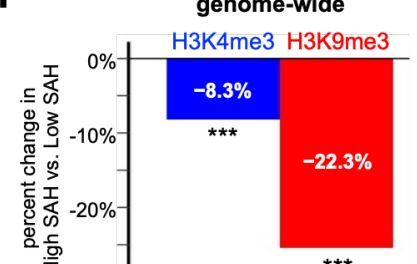

***
H

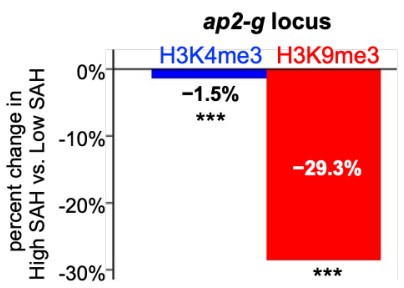

Figure 4. Changes in intracellular SAM and SAH alter the efficiency of heterochromatin maintenance at the ap2-g locus.

(A) Differences in the distribution of H3K4me3 (blue) and H3K9me3 (red) between parasites grown in low SAM (-cho, -met) versus high SAM (+cho, +met) conditions as determined by CUT\& RUN. The intracellular SAM and SAH abundances are shown in the top right. The box on chromosome 12 indicates location of the ap2- $g$ locus. Red bars indicate regions of heterochromatin under high SAM conditions. $n=2$. (B) Mean genome-wide change in H3K4me3 (blue) and H3K9me3 (red) coverage in low SAM versus high SAM conditions. (C) Changes in the distribution of H3K4me3 (blue) and H3K9me3 (red) at the ap2-g locus under conditions between parasites grown in low SAM (-cho, -met) versus high SAM (+cho, +met) conditions. (D) Mean change in coverage across the ap2-g heterochromatin peak (red bar) of H3K4me3 (blue) and H3K9me3 (red) between parasites grown in low SAM versus high SAM conditions. (E) Genome-wide differences in the distribution of H3K4me3 (blue) and H3K9me3 (red) between parasites grown in high SAH (+cho, +3-DZA) versus low SAH (+cho) condition as determined by CUT\& RUN. The box on chromosome 12 indicates location of the ap2-g locus. Red bars indicate regions of heterochromatin under high SAM conditions. $n=3$ (F) Mean genome-wide change in H3K4me3 (blue) and H3K9me3 (red) coverage in high SAH versus low SAH conditions. (G) Differences in the distribution of H3K4me3 (blue) and H3K9me3 (red) between parasites grown in high SAH versus low SAH conditions. (H) Mean change in coverage across the ap2-g heterochromatin peak (red bar) of H3K4me3 (blue) and H3K9me3 (red) between parasites grown in high SAH versus low SAH conditions. 
Similarly, we evaluated the effects of high versus low intracellular SAH levels by growing parasites in the presence of abundant choline with $100 \mathrm{nM}$ 3-DZA added to the culture medium (high SAH) versus abundant choline alone (low SAH) (Figure 3K). Like parasites grown with excess choline and methionine, those grown with high levels of intracellular SAH saw substantial reductions in H3K9me3 occupancy, with H3K4me3 being less responsive (Figure 4E-F). Thus, $\mathrm{H} 3 \mathrm{~K} 9 \mathrm{me} 3$ abundance is more responsive to changes in SAM metabolism, and in such a way that positively correlates to parasite levels of SAM, and negatively correlates to parasite SAH.

When we looked specifically at the pfap2-g locus on chromosome 12, under conditions of low SAM abundance or high intracellular SAH, we observed a significant decrease in H3K9me3 occupancy across the locus, including the promoter region containing the PfAP2-G binding sites that drive the transcriptional feedback loop (Figure 4C-D, 4H-G). These data demonstrate that $\mathrm{H} 3 \mathrm{~K} 9 \mathrm{me} 3$ occupancy at the pfap2-g locus is responsive to changes in SAM and SAH. Under conditions of choline and methionine depletion, cellular pools of SAM are less available in the nucleus to serve as a methyl donor for H3K9me3 placement at the pfap2-g locus, enhancing sexual commitment (Figure 1C, 3C). Similarly, increased levels of SAH additionally impair the placement of H3K9me3 at the pfap2-g locus, further increasing sexual commitment (Figure 3L).

\section{DISCUSSION}

Malaria parasites have evolved sophisticated mechanisms to sense and adapt to the diverse physiological niches they occupy during their life cycle. The switch from asexually replicating blood stage parasites to male and female gametocytes requires balancing a trade-off between maintaining in-host persistence and maximizing transmission between hosts. Several metabolites have been implicated in affecting this switch. Serine and homocysteine both increase frequencies of sexual differentiation(18, 30), while lysoPC and its metabolic product, choline, both act as potent suppressors of sexual commitment(14). Additionally, the concentrations of these precursors are especially low in the bone marrow, the primary site of P. falciparum gametocytogenesis\{Joice:2014bj, Brancucci:2017cx\}. Without the ability to cytoadhere to vascular endothelium, maturation in the bone marrow protects immature gametocytes from splenic clearance by keeping them out of circulation until prior to maturation into highly deformable stage $\mathrm{V}$ gametocytes that are able to clear the spleen\{Tiburcio:2012fl, Neveu:2020ik\}. Here we show that the methyl donor SAM and its metabolic product SAH serve as intermediates that integrate lysoPC availability into changes in H3K9me3 maintenance both genome-wide and specifically at the pfap2-g locus, resulting in altered gene expression leading to changes in sexual commitment rates. Our data demonstrate that the effects of lysoPC on PfPMT activity results in substantial changes to the cellular availability of SAM and SAH, allowing parasites to increase sexual differentiation in the bone marrow and other sites of reduced lysoPC availability. 
In asexual parasites pfap2-g, the master regulator of sexual commitment, is silenced by H3K9me3mediated HP1 binding at the locus. Thus, gametocyte formation rates are influenced by changes in the epigenetic landscape. We propose a model by which the regulatory effect of phospholipid precursors on the initiation of sexual differentiation is mediated by effect of de novo PtdCho synthesis on parasite SAM and SAH availability via PfPMT activity. This model provides a molecular mechanism explaining the previously reported effects of P-cho precursors(14), serine(18), and homocysteine(30) on sexual commitment in P. falciparum, as well as explaining why sexual commitment in rodent parasites is non-responsive to P-cho precursors. Reduced PfPMT activity reduced the burden on parasite SAM pools for PtdCho synthesis and resulted in elevated SAM abundance and/or a decrease in inhibitory SAH levels, making methyl groups more available for histone methyltransferase activity.

The effect of SAM metabolism on DNA methylation has been described in several systems, bridging nutrition, metabolism, and epigenetics. Since SAM is used for a host of protein and nucleotide methylation, it serves as a critical link between metabolism and gene expression. In budding yeast and most other eukaryotes, PtdCho is generated by direct methylation of phosphatidylethanolamine and is a major consumer of SAM and producer of SAH that governs methylation of histones as well as the phosphatase PP2A(22, 34). Unlike most eukaryotes, PfPMT methylates phospho-ethanolamine to make P-cho for the synthesis of PtdCho instead of methylating PtdEtn. However, like yeast PfPMT is also a substantial drain of SAM and source of SAH. We found that the suppression of PfPMT activity by P-cho precursors translated to changes in histone methylation under conditions of low versus high intracellular SAM demonstrated that H3K9me3 is more sensitive to changes in SAM abundance suggesting that the methyltransferase depositing this mark, likely PfSET3, possesses a $K_{m}$ in the range of the intracellular SAM concentrations under these experimental conditions (Figure 4A-B). Under conditions of low cellular SAM availability, H3K9me3 is depleted at every position of occupancy genome wide. Importantly, H3K9me3 is depleted across the pfap2-g locus, including at the region of the promoter containing the PfAP2-G binding sites that drive the transcriptional feedback loop. Thus, under conditions of depleted P-cho precursors and reduced SAM availability for trimethylation of $\mathrm{H} 3 \mathrm{~K} 9$, heterochromatin is depleted at the ap2-g locus, allowing for increased accessibility to proteins that promote transcription, including PfAP2-G itself, explaining the increase in the frequency of sexual differentiation (Figure 4C-D). Furthermore, increasing feedback inhibition of methyltransferases by SAH by blocking SAHH activity also resulted in a genome wide depletion of H3K9me3 but had substantially fewer profound effects on H3K4me3 levels. Since the sensitivity of product inhibition of lysine methyltransferases by SAH varies over a broad range(38), this suggests that the $K_{D}$ of PfSET3 falls within the range of SAH concentrations while the $K_{D}$ of methyltransferases depositing the H3K4me3 mark (PfSET1, PfSET10) are likely higher. 
In summary, changing availability of host lysoPC results in a shift in parasite SAM/SAH ratio which leads to changes in histone methylation, specifically $\mathrm{H} 3 \mathrm{~K} 9 \mathrm{me} 3$, and consequentially rates of sexual commitment in P. falciparum. This finding represents the first characterization of the mechanism by which lysoPC abundance triggers a cascade of events that result in pfap2-g activation and downstream sexual commitment.

\section{ACKNOWLEDGMENTS}

General: We wish to thank Dr. Choukri Ben Mamoun (Yale School of Medicine) for the gift of the pfpmt knockout and complemented lines and the Weill Cornell Medicine genomics core for technical support.

Funding: This work was supported by funds from Weill Cornell Medicine (BK), NIH 1R01 AI141965 (BK), NIH 1R01 Al138499 (KWD), NIH 5F31Al136405-03 (CH), NIH R25 Al140472 (KYR), the Fundação para a Ciência e Tecnologia (MMM, DRIVER-LISBOA-01-0145-FEDER-030751) and “laCaixa" Foundation (MMM, under the agreement HR17/52150010).

Author contributions: Conceptualization: BFCK, KWD, MMM; Methodology: BFCK, CTH, MMM; Investigation: CTH, XT, LNV, IMM; Software, Formal Analysis, Data Curation: BFCK, CTH, XT; Writing - Original Draft: CTH Writing - Review \& Editing: BFCK, CTH, MMM; Visualization: BFCK, CTH; Supervision: BFCK, KYR, MMM; Project Administration: B.F.C.K; Funding Acquisition: BFCK, KWD

Competing interests: The authors declare no competing interests.

Data and materials availability: All data needed to evaluate the conclusions in the paper are present in the paper or the Supplementary Materials. Cut \& Run ChIP-seq data can be obtained from the NCBI Gene Expression Omnibus (GSEXXXX). Cut \& Run analysis code is available at https://github.com/KafsackLab/XXX. 


\section{MATERIALS AND METHODS}

\section{P. falciparum cell culture}

The parasite strains used for this study were NF54 obtained from BEI Resources and the Pf-peg4-tdTomato (39). Cultures were maintained using established culturing techniques (40). Standard complete media was RPMI-1640 supplemented with 25 mM HEPES, $368 \mu$ M hypoxanthine, 1 mM sodium hydroxide, 24 mM sodium bicarbonate, 21 MM gentamycin (Millipore Sigma), with Albumax II Lipid-Rich BSA (Gibco), unless otherwise stated. Concentrations for choline and lysoPC supplementation were used according to (14). See Supplementary Table 1 for changes to media compositions used throughout this manuscript. RBCs or cultures were washed three times with respective experimental media conditions at the start of each change in media condition.

\section{Generation of PfSAMS-gImS and PfPMT-gImS conditional knockdown lines}

The 3' portion of the coding sequence for pfsams (577-1206bp) and pfpmt (651-1217bp) was amplified from gDNA and cloned by Gibson assembly into the pSLI-HAx3-gImS plasmid (gift from Professor R. Dzikowski) (25) following Notl and Xmal double digest. Following transfection of NF54 parasites, cultures were selected for the presence of the plasmid with 4nM WR99210 (gift of Jacobus Pharmaceuticals), follow by selection for integrants with G418 (Millipore Sigma). Single-crossover integration of the plasmid was confirmed via PtdChoR (Figure S3 \& S5), and parasites were cloned to isolate a population with single cross-over lacking any remaining WT loci. Integrated parasite lines were maintained under G418 drug pressure.

\section{Animal Maintenance}

Animal research was conducted at the Instituto de Medicina Molecular (Lisboa, Portugal). All protocols were approved by the animal ethics committee (ORBEA committee) at the institute and performed according to national and European regulations. BALB/c mice (age 5-8 weeks; males) were purchased from Charles River Laboratories (Saint-Germain-sur-l'Arbresle, France), kept in specific-pathogen-free conditions, and subjected to regular pathogen monitoring by sentinel screening. Experimental animals were randomly assigned and allowed free access to water and food.

\section{Generation of PbSAMS-DD conditional knockdown parasites}

Wild-type $P$. berghei ANKA strain was obtained from the MR4 repository (Manassas, Virginia). P. berghei pbsams-DD parasite line was obtained by double crossover homologous recombination. To do so, parasites were transfected by electroporation of purified schizonts, harvested on day 7-10 post transfection and genotyped by 
PtdChoR. Transgenic parasites were then dilution cloned and further stored at $-80{ }^{\circ} \mathrm{C}$ in frozen blood vials, containing $10^{7}$ blood stage parasites.

Recombinant parasites carrying the human dihydrofolate reductase ( $h d h f r)$ gene cassette were positively selected by treatment of mice with pyrimethamine and trimethoprim to stabilize PbSAMS. Confirmation of transgenic parasite genotype, construct integration at the desired genomic loci and elimination of WT locus were assessed by PtdChoR. Blood from the tail vein of infected mice was collected in $200 \mu \mathrm{L}$ of $1 \mathrm{x}$ PBS and genomic DNA was isolated using the NZY Blood gDNA Isolation Kit (NZYTech), according to manufacturer's guidelines. Stabilization of PbSAMS-DD fusion protein throughout infection was achieved in vivo, by administration of trimethoprim (TMP) to mice $(0.25 \mathrm{mg} / \mathrm{ml}$ of TMP in drinking water), 2 days prior to infection.

\section{Quantification of PbSAMS knockdown}

All steps of parasite pellet extraction protocol were performed at 4 으 $\mathrm{C}$ to minimize protein degradation and all centrifugations were executed at 1000 x g for $10 \mathrm{~min}$. Mice were sacrificed at day 4 post infection, $1 \mathrm{~mL}$ of blood was collected by cardiac punction, washed in $10 \mathrm{~mL}$ of $1 \mathrm{x}$ PBS and centrifuged. Packed erythrocytes were saponin-lysed in $0.15 \%$ saponin and centrifuged. Parasite pellet was washed twice in PBS containing $1 x$ Proteinase inhibitor cocktail (Roche cOmplete Protease inhibitor tablets, EDTA free). Parasite pellet was then resuspended in lysis buffer (4 \% SDS; $0.5 \%$ Triton X-114 in 1x PBS), incubated on ice for 10 min, centrifuged at $21000 \mathrm{x} g$ for $10 \mathrm{~min}$ and the supernatant was collected. Total protein content was determined using the BioRad protein assay kit according to manufacturer's instructions. Protein samples diluted in 5x SDS sample buffer (NZYTech) were denatured at 95의 for $10 \mathrm{~min}$ and resolved in an 8\% polyacrylamide gel (SDS-PAGE). Proteins were blotted into a nitrocellulose membrane by wet transfer at 200mA for 2 hours. Primary antibodies, mouse anti-HA antibody (1:1000, from Covance) and rabbit anti-Bip (1:2000, GeneScript) were incubated overnight at 4으. Secondary antibodies, anti-mouse horseradish peroxidase (HRP)-conjugated and anti-rabbit HRP (1:10000, Jackson ImmunoResearch Laboratories) were incubated at RT for 1 hour. Signal detection was obtained using Luminata Crescendo Western HRP substrate (Merck Milipore ${ }^{\circledR}$ ) and the ChemiDoc XRS+ Gel Imaging System $\left(\right.$ BioRad $\left.^{\circledR}\right)$. Protein band quantification was performed on Image Lab software (version 5.0) using BIP levels for normalization.

\section{Quantification of gametocytes in PbSAMS-DD-HA parasites}

Mice infections were performed by intraperitoneal inoculation of $1 \times 10^{6}$ infected red blood cells (iRBCs) obtained by passage in the correspondent BALB/c background mice. Parasitemia (\% of iRBCs) was monitored daily and gametocytemia (\% of mature gametocytes) was determined on day 3 after infection, by microscopic analysis of 
Giemsa-stained blood smears. A total of 5-10 thousand RBCs were analysed in randomly acquired images and semi-automatically quantified using Image J software (http://rsbweb.nih.gov/ij).

\section{Induction and quantification of $P$. falciparum sexual commitment}

Synchronous gametocyte induction was performed as previously described (9). Briefly, parasites were doublesynchronized with $5 \%$ sorbitol to achieve a synchrony of $\pm 6 \mathrm{~h}$ in the previous cycle. Synchronized ring-stage parasites were set up at $1.5 \%$ parasitemia (1\% hematocrit) in 96-well, flat bottom plates under specific nutrient conditions to induce sexual commitment. Following reinvasion, on the first day of gametocyte development $(D+1)$, ring-stage parasitemia was determined using flow cytometry and 50mM N-acetyl-D-glucosamine was added for 5 consecutive days. Gametocytes were then counted on day $6(D+6)$ and commitment rate was determined by dividing the $D+6$ gametocytemia by the $D+1$ parasitemia assessed prior to $N$-acetyl-Dglucosamine addition. Gametocytes induced using the Pf-peg4-tdTomato fluorescent gametocyte reporter line were counted using flow cytometry (39).

\section{Metabolite Analysis}

Synchronous $P$. falciparum parasites were grown from 8 hpi to $34 \mathrm{hpi} \pm 6 \mathrm{hpi}(100 \mathrm{~mL}, 3 \%$ hematocrit, $5-6 \%$ parasitemia) under the indicated growth conditions. Infected red blood cells (iRBC) were then purified to $>90 \%$ purity using a 70/40\% percoll/sorbitol density gradient and centrifugation (4700 G for 15 minutes at room temperature), then washed three times with minimal RPMI. Following isolation, equal numbers of isolated iRBC, as determined by the Beckman Coulter Z1 Coulter Particle Counter, were then re-incubated in their respective treatment conditions for four more hours to allow parasites to recover from percoll/sorbitol isolation. Following recovery, the purity of iRBCs was assessed using flow cytometry, cells were then pelleted, washed once with 1 $\mathrm{mL}$ of $1 x$ PBS, and quickly lysed with $500 \mu \mathrm{L}$ of $90 \%$ ultrapure HPLC Grade methanol (VWR), followed by exactly 10 seconds of vortexing before being stored in dry ice. Lysed samples were then centrifuged at top speed at $4^{\circ} \mathrm{C}$ and supernatant collected for metabolite analysis. Uninfected red blood cells (URBC) were incubated under the same treatment conditions as iRBC, counted, and methanol extracted to distinguish iRBC metabolite signal from URBC signal. Extracted metabolites were stored at $-80^{\circ} \mathrm{C}$. LC-MS based metabolomic analysis was used as previously described (41). $10 \mu \mathrm{L}$ of extract was separated on an Agilent 1290 Infinity LC system. Acquisition was performed on an Agilent 6230 TOF mass spectrometer (Agilent Technologies, Santa Clara, CA) employing an Agilent Jet Stream electrospray ionization source (Agilent Technologies, Santa Clara, CA) operated in high resolution, positive mode. Metabolite identification was verified by exact mass (to $35 \mathrm{ppm}$ ) and co-elution with authentic standards purchased from Millipore Sigma. Batch feature extraction and chromatographic alignment 
across multiple data files was performed using the Agilent MassHunter Profinder software and extracted metabolite data was exported for further statistical analysis using R.

\section{Western Blotting}

PfPMT-gImS and PfSAMS-gImS knockdown western blots were performed using whole cell lysate. Ring-stage parasites (12hpi $\pm 6 \mathrm{hpi}$ ) were treated with or without $2.5 \mathrm{mM}$ glucosamine (VWR) to induce protein knockdown as previously described. Parasites were saponin lysed at 36hpi $\pm 6 \mathrm{hpi}$ and whole cell extract collected in SDS sample buffer, then boiled for 10 minutes. Proteins were then separated on $12 \%$ SDS PAGE and transferred to a PVDF membrane (Millipore Sigma, $0.2 \mu \mathrm{M}$ ). Membranes blocked with 5\% milk were then probed with anti-HA (1:2000, Abcam, ab9110), and anti-hsp70 (1:2000, StressMarq SPtdCho-186) primary antibody solutions, followed by anti-rabbit IgG (1:5000, Millipore Sigma 12-348) secondary. Chemiluminescence was measured using the Azure c-Series imaging systems (Azure Biosystems) and quantified using the Fiji open-source image processing package based on ImageJ.

\section{Quantification of changes in H3K9me3 and H3K4me3 abundance and distribution.}

H3K9me3 and H3K4me3 abundances were measured by Cleavage Under Targets \& Release Using Nuclease (CUT \& RUN) (42) adapted for $P$. falciparum. Approximately $1 \times 10^{7}$ percoll/sorbitol isolated schizonts (34-38 hpi) per sample were washed three times with $1 \mathrm{ml}$ wash buffer $(20 \mathrm{mM} \mathrm{HEPES}$ at $\mathrm{pH} 7.5,150 \mathrm{mM} \mathrm{NaCl}, 0.5 \mathrm{mM}$ Spermidine, and $1 \times$ Roche complete protease inhibitor) at room temperature and resuspended in $225 \mathrm{ul}$ of wash buffer. 25ul of Concanavalin A-coated beads were washed and resuspended in binding buffer (20 mM HEPES$\mathrm{KOH}$ at pH 7.5, $10 \mathrm{mM} \mathrm{KCl}, 1 \mathrm{mM} \mathrm{CaCl}$, $1 \mathrm{mM} \mathrm{MnCl2)}$ before being added to each sample. Samples were then rotated for 10 minutes at room temperature. Then, samples were placed on a magnetic stand to clear (30s to 2 minutes) and remove all the liquid. Samples were washed 3x's with DIG wash buffer (wash buffer with $0.025 \%$ digitonin). For each sample, $150 \mu$ l of antibody wash buffer was added (wash buffer, $0.025 \%$ digitonin, and $2 \mathrm{mM}$ EDTA at pH 8.0) with H3K4me3 $(0.005 \mu \mathrm{g} / \mu \mathrm{l}$, C15410003-50, Diagenode), H3K9me3 (0.005 $\mu \mathrm{g} / \mu \mathrm{l}$, ab8898, abcam), or IgG isotype control $(0.005 \mu \mathrm{g} / \mu \mathrm{l}, 02-6102$, ThermoFisher) was added to the sample tube and incubated at $4{ }^{\circ} \mathrm{C}$, rotating, overnight. Following antibody incubation, samples were placed on a magnetic stand to clear and pull off the liquid, then washed 3 x's with DIG wash buffer, $150 \mu$ of ProteinA/G-MNASE fusion protein (dilution 1:60, 15-1016, EpiCypher) in DIG wash buffer and rotated at $4{ }^{\circ} \mathrm{C}$ for 1 hour. After washing samples 3x's with DIG wash buffer, samples were then washed 3x's with Low Salt Rinse Buffer (20 mM HEPES$\mathrm{NaOH}$ at pH 7.5, 0.025\% digitonin, $0.5 \mathrm{mM}$ spermidine). $200 \mu \mathrm{l}$ of ice-cold incubation buffer (3.5 mM HEPES$\mathrm{NaOH}$ at pH 7.5, $100 \mathrm{mM} \mathrm{CaCl}_{2}, 0.025 \%$ DIG) was added and samples were equilibrated at $0{ }^{\circ} \mathrm{C}$ for 30 minutes to achieve targeted digestion. Digestion was then stopped by adding $200 \mu \mathrm{l}$ of stop buffer (170 mM NaCl, $20 \mathrm{mM}$ 
EGTA at pH 8.0, $20 \mathrm{mM}$ EGTA, 0.0.25\% digitonin, $25 \mu \mathrm{g} / \mathrm{mL}$ glycogen, $50 \mu \mathrm{g} / \mathrm{mL}$ RNase A). After incubation at 37 ${ }^{\circ} \mathrm{C}$ for 30 minutes to release soluble fragments, samples were digested by adding $2.5 \mu \mathrm{L}$ proteinase $\mathrm{K}(20 \mathrm{mg} / \mathrm{ml})$ and $2 \mu \mathrm{L} 10 \% \mathrm{SDS}$, and then incubated at $50{ }^{\circ} \mathrm{C}$ for 1 hour. DNA fragments were purified with phenolchloroform-isoamyl alcohol and washed by ethanol precipitation, and finally dissolved in $30 \mu \mathrm{L}$ TE buffer (1mM Tris-HCl pH 8, 0.1 mM EDTA).

Library construction was carried out using NEBNext Ultra II DNA Library Prep Kit for Illumina (E7645S, NEB). 4ng of fragmented DNA was used for end-repair/A-tailing, ligation, and post ligation cleanup with 1.7x volumes of AMPure XP beads (Catalog number, company). Following cleanup, PtdChoR amplification was performed using 2x KAPA HotStart ready mix (Catalog number, company) and NETFLEX primer mix (Catalog number, Bio Scientific) with PtdChoR program: 1 min @ 98 $\mathrm{C} / 15$ cycles: $10 \mathrm{sec} @ 98^{\circ} \mathrm{C} / 1 \mathrm{~min} @ 65^{\circ} \mathrm{C} / / 5 \mathrm{~min}$ @ $65^{\circ} \mathrm{C} / /$ hold $4^{\circ} \mathrm{C}$. PtdChoR products were size selected with $0.8 \mathrm{x}$ volumes, then $1.2 \mathrm{x}$ volumes of AMPure XP beads. Beads were then washed twice with $80 \%$ ethanol and DNA eluted with $0.1 \mathrm{x}$ TE and used for sequencing in a Nextseq2000 system as paired-end reads, following quality control.

After sequencing, raw reads were trimmed using Trimmomatic v0.38 (43) to remove residual adapter sequences and low quality leading and trailing bases. Both paired and unpaired reads were retained for read lengths that were at least 30 bases after trimming. Trimmed paired reads were aligned to the PlasmoDB version 46 P. falciparum 3D7 reference genome (26) using BWA v.0.7.1 (44). SAMtools v.1.10 (45) was used to remove low quality alignments as well as sort and index sample files. Normalized fold enrichment tracks were generated by using the MACS2 v.2.2.7.1 (46) callpeak function with settings: -f BAMPE -B -g 2.3e7 -q 0.05 -nomodel -broad -keep-dup auto -max gap 500. Bedgraph outputs were then passed into the bdgcmp function with the setting -m FE (fold enrichment) to generate signal tracks to profile histone modification enrichment levels compared to whole genome. Peak sets from replicates were compared with Bedtools intersect v2.26.0 (47), and peaks that overlapped by at least $1 \mathrm{bp}$ were considered shared. Fold enrichment bedgraphs and peak sets were then output to RStudio Server (v1.4.1717) for further analysis using the GenomicRanges Package v.1.44.0 (48) and visualized with the GViz v1.38.1 package (49) within the Bioconductor project (release 3.13) (50). 


\section{REFERENCES}

1. C. Drakeley, C. Sutherland, J. T. Bousema, R. W. Sauerwein, G. A. T. Targett, The epidemiology of Plasmodium falciparum gametocytes: weapons of mass dispersion. Trends in Parasitology. 22, 424-430 (2006).

2. B. F. C. Kafsack et al., A transcriptional switch underlies commitment to sexual development in malaria parasites. Nature. 507, 248-252 (2014).

3. A. Sinha et al., A cascade of DNA-binding proteins for sexual commitment and development in Plasmodium. Nature. 507, 253-257 (2014).

4. J. J. Lopez-Rubio, L. Mancio-Silva, A. Scherf, Genome-wide analysis of heterochromatin associates clonally variant gene regulation with perinuclear repressive centers in malaria parasites. Cell Host Microbe. 5, 179-190 (2009).

5. N. M. B. Brancucci et al., Heterochromatin protein 1 secures survival and transmission of malaria parasites. Cell Host Microbe. 16, 165-176 (2014).

6. S. A. Fraschka et al., Comparative Heterochromatin Profiling Reveals Conserved and Unique Epigenome Signatures Linked to Adaptation and Development of Malaria Parasites. Cell Host Microbe. 23, 407420.e8 (2018).

7. M. Filarsky et al., GDV1 induces sexual commitment of malaria parasites by antagonizing HP1dependent gene silencing. Science. 359, 1259-1263 (2018).

8. A. J. Bannister et al., Selective recognition of methylated lysine 9 on histone H3 by the HP1 chromo domain. Nature. 410, 120-124 (2001).

9. A. Poran et al., Single-cell RNA sequencing reveals a signature of sexual commitment in malaria parasites. Nature. 551, 95-99 (2017).

10. G. A. Josling et al., Dissecting the role of PfAP2-G in malaria gametocytogenesis. Nature Communications. 11, 1-13 (2020).

11. O. Llorà-Batlle et al., Conditional expression of PfAP2-G for controlled massive sexual conversion in Plasmodium falciparum. Science advances. 6, eaaz5057 (2020).

12. R. S. Kent et al., Inducible developmental reprogramming redefines commitment to sexual development in the malaria parasite Plasmodium berghei. Nat. Microbiol. 3, 1206-1213 (2018).

13. G. Neveu, D. Beri, B. F. Kafsack, Metabolic regulation of sexual commitment in Plasmodium falciparum. Curr Opin Microbiol. 58, 93-98 (2020).

14. N. M. B. Brancucci et al., Lysophosphatidylcholine Regulates Sexual Stage Differentiation in the Human Malaria Parasite Plasmodium falciparum. Cell. 171, 1532-1544.e15 (2017).

15. L. C. Pollitt et al., Competition and the evolution of reproductive restraint in malaria parasites. The American Naturalist. 177, 358-367 (2011).

16. R. Joice et al., Plasmodium falciparum transmission stages accumulate in the human bone marrow. Science Translational Medicine. 6, 244re5-244re5 (2014). 
17. K. Venugopal, F. Hentzschel, G. Valkiūnas, M. Marti, Plasmodium asexual growth and sexual development in the haematopoietic niche of the host. Nat Rev Micro. 18, 177-189 (2020).

18. S. Gulati et al., Profiling the Essential Nature of Lipid Metabolism in Asexual Blood and Gametocyte Stages of Plasmodium falciparum. Cell Host Microbe. 18, 371-381 (2015).

19. S. Wein et al., Contribution of the precursors and interplay of the pathways in the phospholipid metabolism of the malaria parasite. J. Lipid Res., jlr.M085589 (2018).

20. N. Kilian, J.-Y. Choi, D. R. Voelker, C. Ben Mamoun, Role of phospholipid synthesis in the development and differentiation of malaria parasites in the blood. Journal of Biological Chemistry. 293, 17308-17316 (2018).

21. A. Garg et al., Structure, function and inhibition of the phosphoethanolamine methyltransferases of the human malaria parasites Plasmodium vivax and Plasmodium knowlesi. Sci. Rep. 5, 9064-13 (2015).

22. C. Ye, B. M. Sutter, Y. Wang, Z. Kuang, B. P. Tu, A Metabolic Function for Phospholipid and Histone Methylation. Molecular Cell. 66, 180-193.e8 (2017).

23. W. H. Witola, C. Ben Mamoun, Choline induces transcriptional repression and proteasomal degradation of the malarial phosphoethanolamine methyltransferase. Eukaryotic Cell. 6, 1618-1624 (2007).

24. W. H. Witola et al., Disruption of the Plasmodium falciparum PfPMT gene results in a complete loss of phosphatidylcholine biosynthesis via the serine-decarboxylase-phosphoethanolaminemethyltransferase pathway and severe growth and survival defects. J Biol Chem. 283, 27636-27643 (2008).

25. P. Prommana et al., Inducible Knockdown of Plasmodium Gene Expression Using the glmS Ribozyme. PLOS ONE. 8, e73783 (2013).

26. S. Warrenfeltz et al., EuPathDB: The Eukaryotic Pathogen Genomics Database Resource. Methods Mol Biol. 1757, 69-113 (2018).

27. R. M. Reguera, C. M. Redondo, Y. Pérez-Pertejo, R. Balaña-Fouce, S-Adenosylmethionine in protozoan parasites: functions, synthesis and regulation. Mol Biochem Parasitol. 152, 1-10 (2007).

28. J. M. Bujnicki, S. T. Prigge, D. Caridha, P. K. Chiang, Structure, evolution, and inhibitor interaction of Sadenosyl-L-homocysteine hydrolase from Plasmodium falciparum. Proteins: Structure, Function, and Bioinformatics. 52, 624-632 (2003).

29. P. K. Chiang, Biological effects of inhibitors of S-adenosylhomocysteine hydrolase. Pharmacol Ther. 77, 115-134 (1998).

30. D. Beri et al., A disrupted transsulphuration pathway results in accumulation of redox metabolites and induction of gametocytogenesis in malaria. Sci. Rep. 7, 40213 (2017).

31. S. J. Mentch et al., Histone Methylation Dynamics and Gene Regulation Occur through the Sensing of One-Carbon Metabolism. Cell Metabolism. 22, 861-873 (2015).

32. S. Li et al., Serine and SAM Responsive Complex SESAME Regulates Histone Modification Crosstalk by Sensing Cellular Metabolism. Molecular Cell. 60, 408-421 (2015). 
33. N. Shyh-Chang et al., Influence of threonine metabolism on S-adenosylmethionine and histone methylation. Science. 339, 222-226 (2013).

34. C. Ye et al., Demethylation of the Protein Phosphatase PP2A Promotes Demethylation of Histones to Enable Their Function as a Methyl Group Sink. Molecular Cell. 73, 1115-1126.e6 (2019).

35. B. M. Sutter, X. Wu, S. Laxman, B. P. Tu, Methionine inhibits autophagy and promotes growth by inducing the SAM-responsive methylation of PP2A. Cell. 154, 403-415 (2013).

36. A. M. Salcedo-Amaya et al., Dynamic histone $\mathrm{H} 3$ epigenome marking during the intraerythrocytic cycle of Plasmodium falciparum. Proceedings of the National Academy of Sciences. 106, 9655-9660 (2009).

37. K. Karmodiya et al., A comprehensive epigenome map of Plasmodium falciparum reveals unique mechanisms of transcriptional regulation and identifies H3K36me2 as a global mark of gene suppression. Epigenetics \& Chromatin. 8, 1150 (2015).

38. M. Luo, Chemical and Biochemical Perspectives of Protein Lysine Methylation. Chem Rev. 118, 66566705 (2018).

39. K. J. McLean et al., Generation of Transmission-Competent Human Malaria Parasites with Chromosomally-Integrated Fluorescent Reporters. Sci. Rep. 9, 1-10 (2019).

40. K. Moll, I. Ljungström, H. Perlmann, A. Scherf, Methods in malaria research. Manassas (2008).

41. E. Ballinger et al., Opposing reactions in coenzyme A metabolism sensitize Mycobacterium tuberculosis to enzyme inhibition. Science. 363 (2019), doi:10.1126/science.aau8959.

42. P. J. Skene, J. G. Henikoff, S. Henikoff, Targeted in situ genome-wide profiling with high efficiency for low cell numbers. Nature Protocols. 13, 1006-1019 (2018).

43. A. M. Bolger, M. Lohse, B. Usadel, Trimmomatic: a flexible trimmer for Illumina sequence data. Bioinformatics. 30, 2114-2120 (2014).

44. H. Li, R. Durbin, Fast and accurate short read alignment with Burrows-Wheeler transform. - PubMed NCBI. Bioinformatics. 25, 1754-1760 (2009).

45. H. Li et al., The Sequence Alignment/Map format and SAMtools. Bioinformatics. 25, 2078-2079 (2009).

46. Y. Zhang et al., Model-based analysis of ChIP-Seq (MACS). Genome Biol. 9, R137 (2008).

47. A. R. Quinlan, I. M. Hall, BEDTools: a flexible suite of utilities for comparing genomic features. Bioinformatics. 26, 841-842 (2010).

48. M. Lawrence et al., Software for Computing and Annotating Genomic Ranges. PLoS Comput. Biol. 9, e1003118 (2013).

49. F. Hahne, R. Ivanek, in Methods in Molecular Biology (2016), vol. 1418, pp. 335-351.

50. W. Huber et al., Orchestrating high-throughput genomic analysis with Bioconductor. Nat Meth. 12, 115121 (2015). 


\section{SUPPLEMENTARY DATA:}

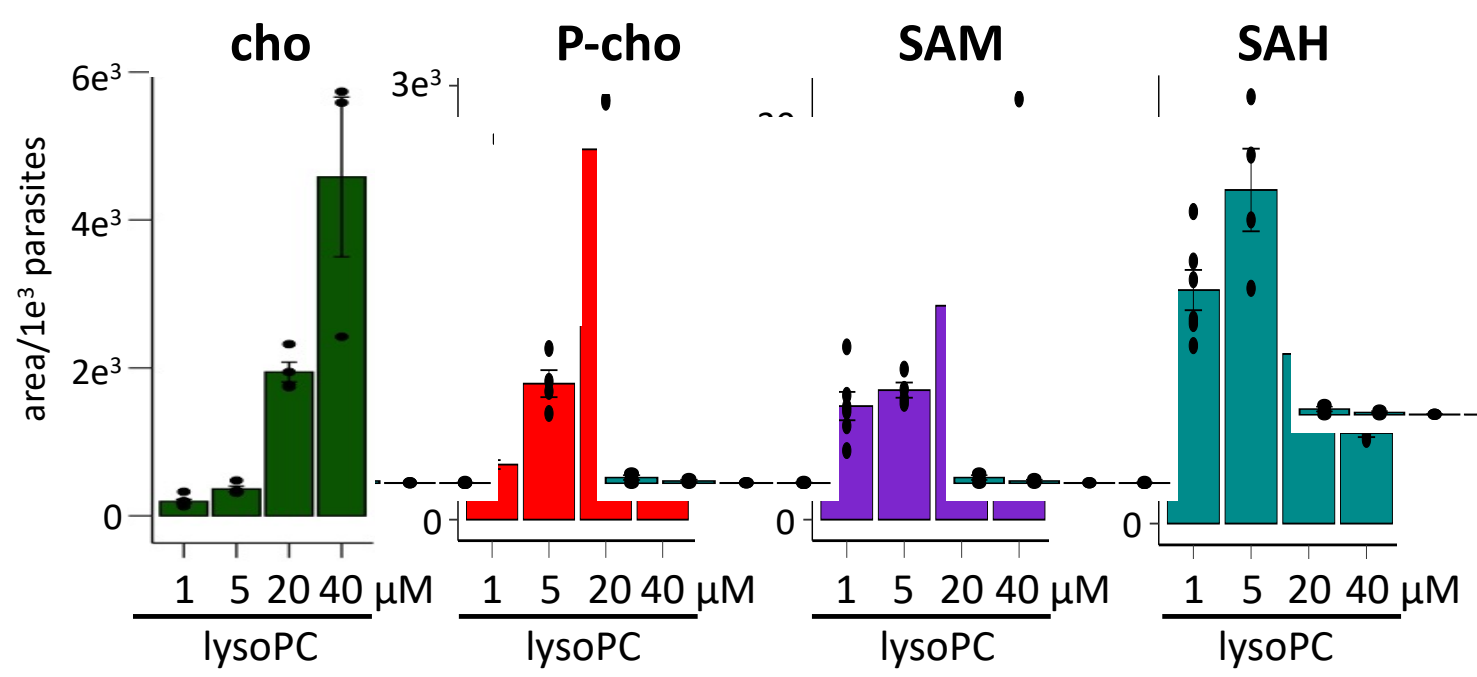

Supplementary Figure S1: Dose-dependent metabolic response to lysoPC.

Parasites were cultured in media spiked with increasing concentrations of lysoPC. Changes in iRBC metabolite levels were assessed by LCMS. Bars quantify intracellular metabolite abundances per thousand parasites $(n=5)$. 

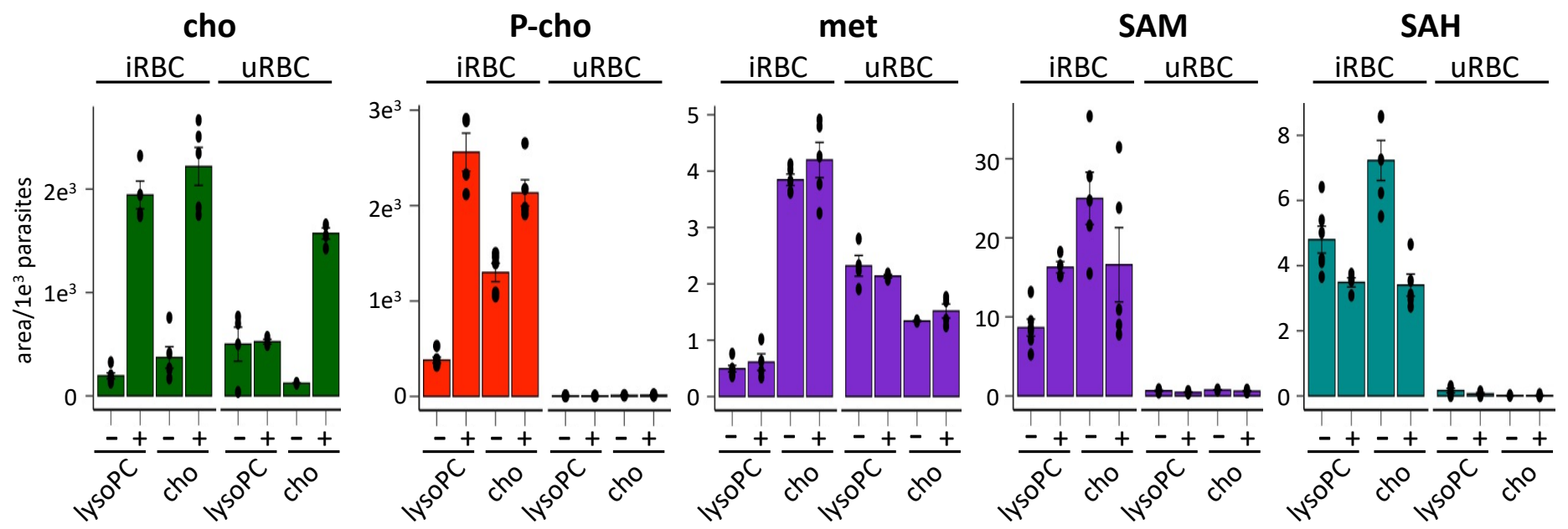

Supplementary Figure S2: Changes in SAM/SAH metabolism are specific to parasite metabolism.

LCMS quantification of indicted metabolites. Infected and uninfected cultures were cultured in the presence or absence of $20 \mu \mathrm{M}$ lysoPC or $420 \mu \mathrm{M}$ choline for $\sim 36 \mathrm{hpi}$ during the commitment cycle. iRBC and uRBC were then extracted simultaneously and metabolite abundances were quantified using LCMS. Bars indicate the area under the curve for each metabolite per thousand parasites. $(n=5)$. 
A

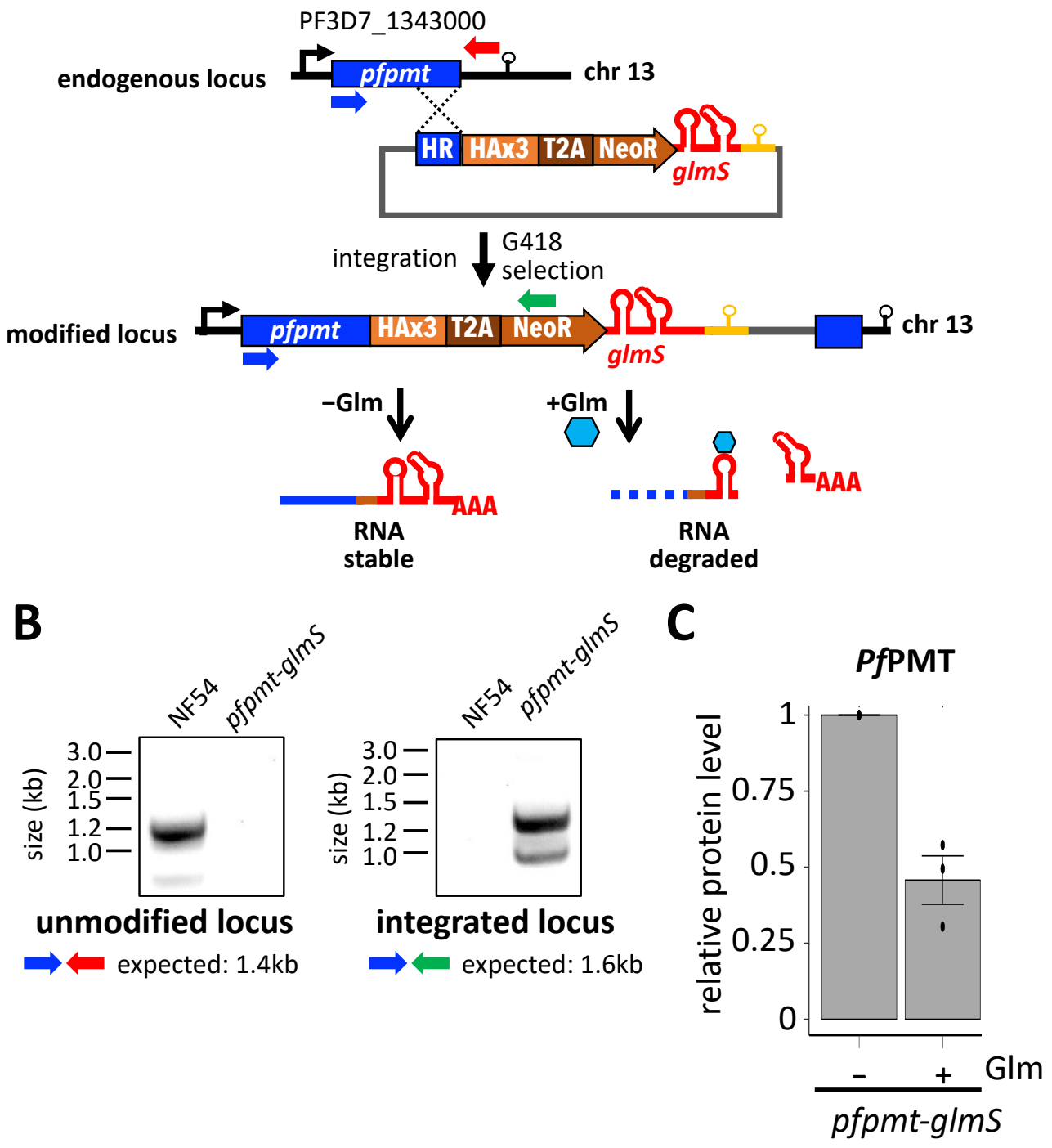

Supplementary Figure S3: Validation of pfpmt-glmS knockdown parasite line.

(A) Generation of pfpmt-glmS knockdown parasites by selection-linked integration. (B) Validation PtdChoR demonstrating tagging of the endogenous pfpmt locus. (C) Western blot densitometry of PfPMT-HAx3 levels in pfpmt-glmS parasites in the presence of $0 \mathrm{mM}(-\mathrm{Glm})$ or $2.5 \mathrm{mM}(+\mathrm{Glm})$ glucosamine relative to Hsp70 loading control $(n=3)$. 


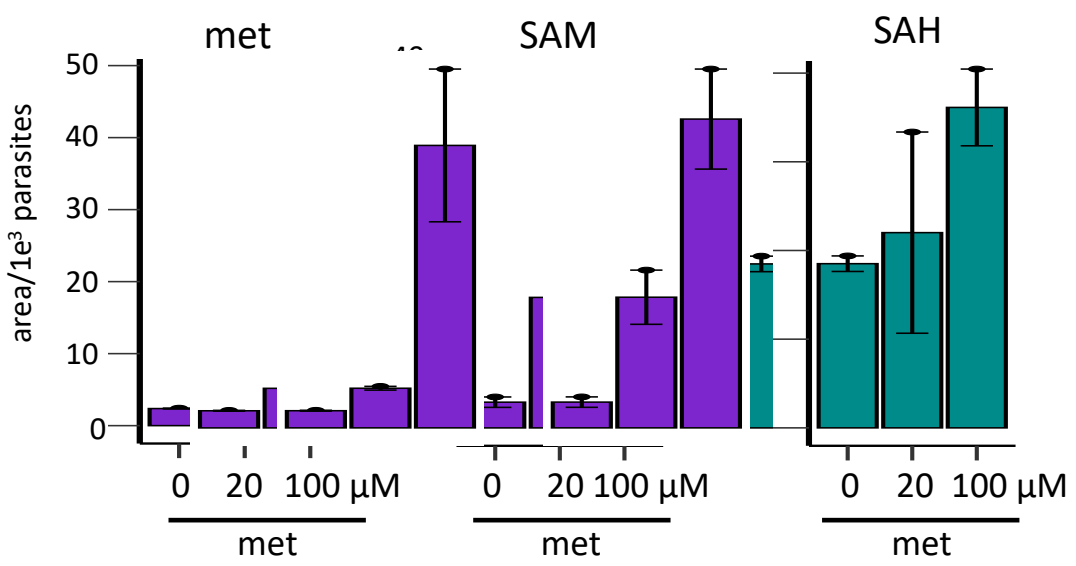

Supplementary Figure S4: Parasite levels of SAM rely heavily on methionine availability in the media.

Parasites were cultured for 34-38 hpi in media spiked with increasing concentrations of methionine before metabolite extraction for LCMS analysis. Bars quantify the area under the curve for each metabolite per thousand parasites $(n=2)$. 
A

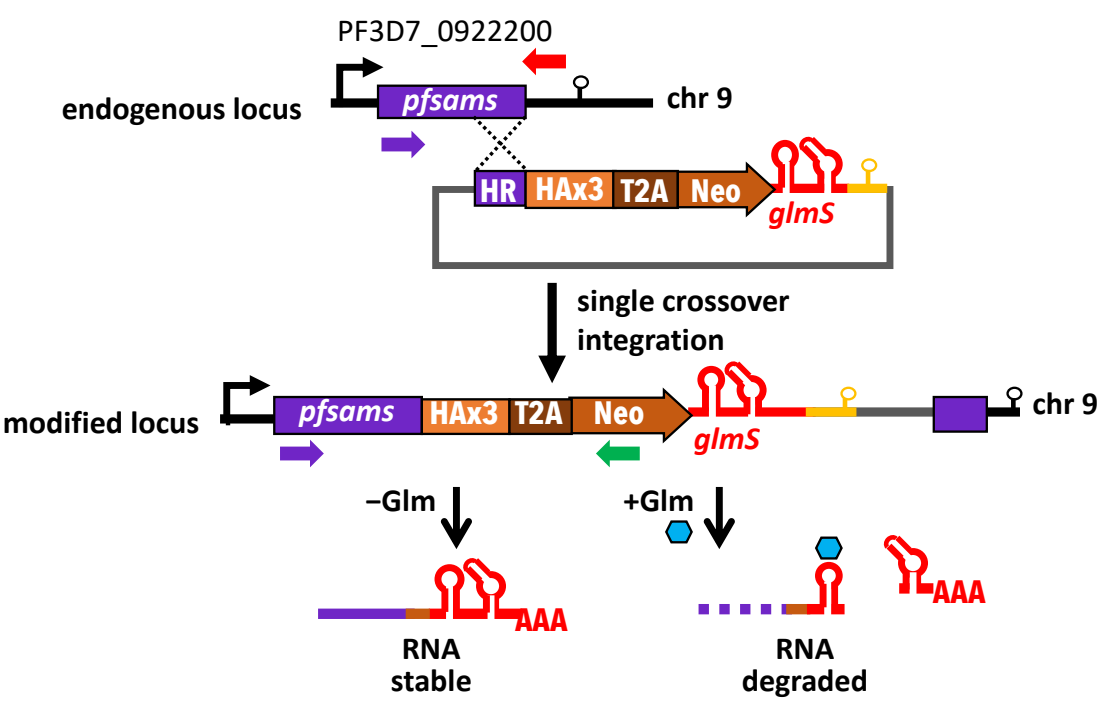

B

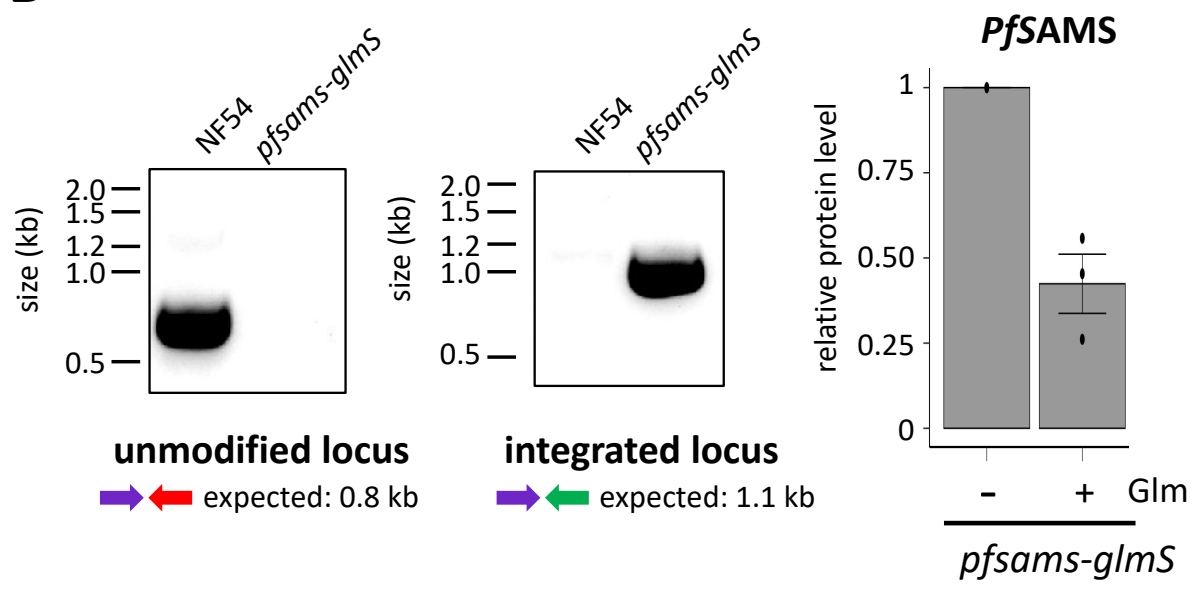

Supplementary Figure S5: Validation of pfsams-glmS knockdown parasite line.

(A) Generation of pfsams-glmS knockdown parasites by selection-linked integration. (B) Validation PtdChoR demonstrating tagging of the endogenous pfsams locus. (C) Western blot densitometry of PfSAMS-HAx3 levels in pfsams-glmS parasites in the presence of $0 \mathrm{mM}(-\mathrm{Glm})$ or $2.5 \mathrm{mM}(+\mathrm{Glm})$ glucosamine relative to Hsp70 loading control $(n=3)$. 
A

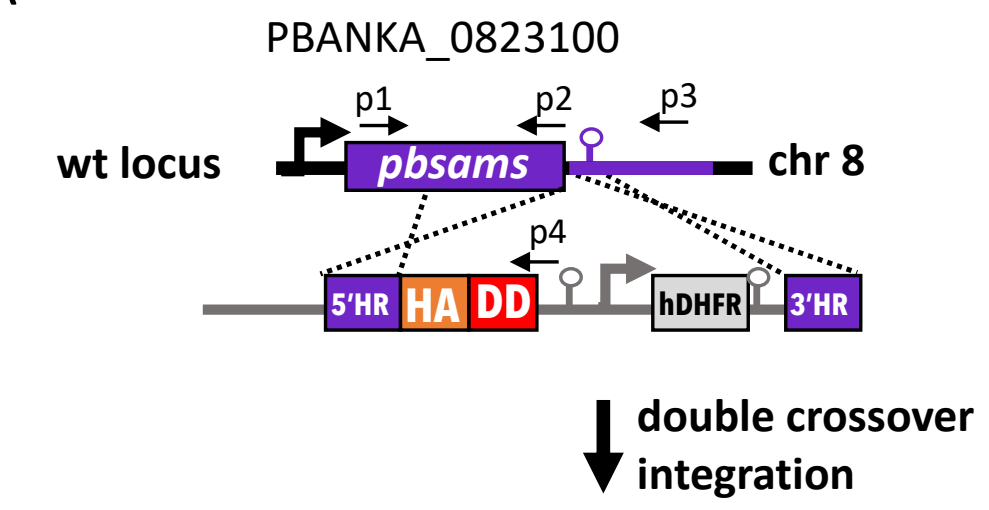

B

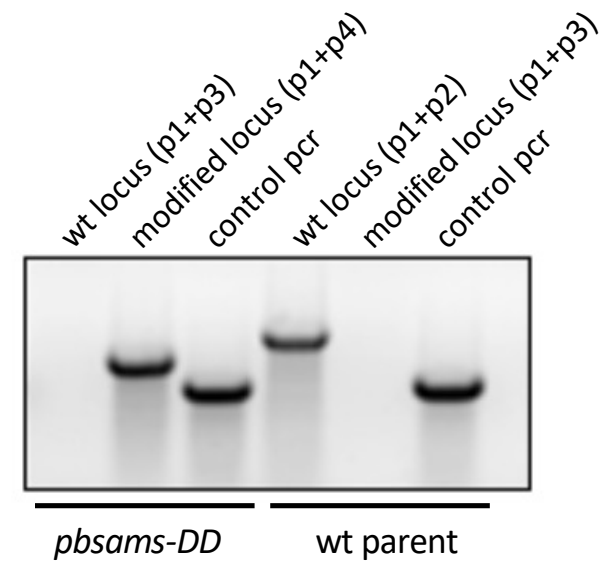

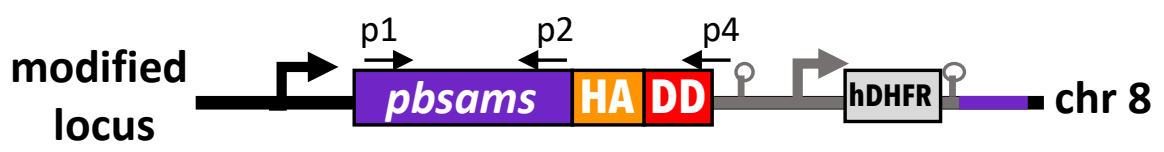

C

pbsams-DD

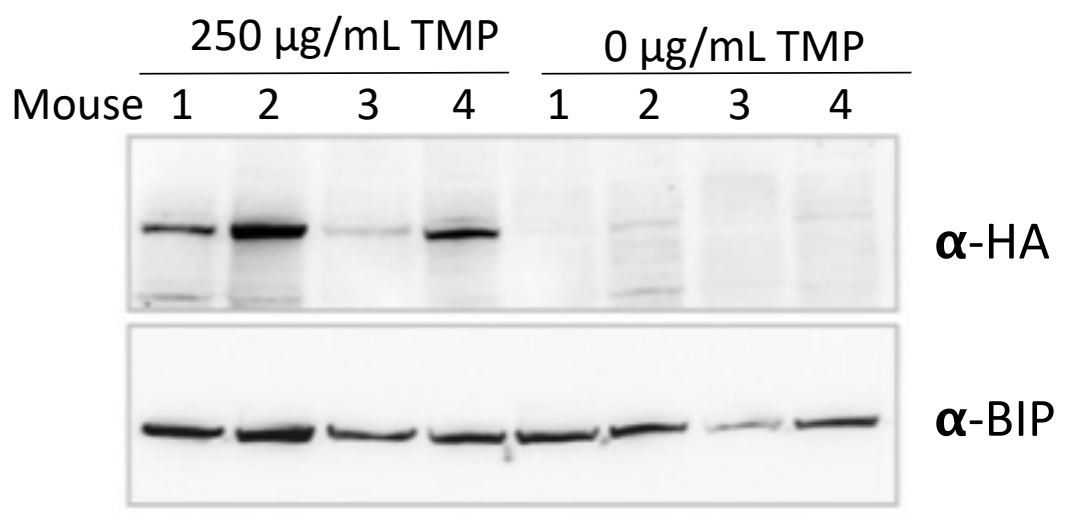

Supplementary Figure S6: Validation of pbsams-DD knockdown parasite line.

(A) The endogenous pbsams locus in the $P$. berghei ANKA strain background was modified by homologous integration to add the ecDHFR destabilization domain (DD) and hemagglutinin epitope tag (HA) at the $3^{\prime}$ end of the pbsams coding sequence. Simultaneous integration of a hDHFR expression cassette allows for selection of integrants. (B) PtdChoR validation of successful tagging in PbSAMS-DD-HA parasites. (C) Successful knockdown of PbSAMS upon removal of trimethoprim (TMP) from the drinking water in mice infected with pbsams-DD parasites. Parasite lysates were assayed for the abundance of PbSAMS-DD with antibodies against the HA epitope tag and $P b B I P$, which served as a loading control and was used for normalization. 